\title{
SUR LES ROUTES DE KIGALI : ÉVOLUTION DES PRATIQUES DE PETITE CORRUPTION
}

La lutte contre la corruption a eu pour effet d'accentuer la privatisation informelle de la gestion du secteur du transport au Rwanda au profit de coopératives qui ont remplacé la police routière dans les opérations de contrôle. Cette imbrication du formel et de l'informel facilite le détournement des règles relatives à l'ordre sur la voie publique et I'instrumentalisation de l'autorité de l'État. La corruption est ainsi facilitée par un manque de contrôle des agents de sécurité, de multiples rapports de domination à l'encontre des taxis-motos et les jeux de pouvoir au sein des coopératives, et entre celles-ci et les agents de police. Le contexte autoritaire du Rwanda mène finalement à une application partiale des règles, sans que cette partialité puisse être contestée puisqu'elle fait partie des rapports de force existants. Si le contraste entre l'image du Rwanda et la réalité empirique est saisissant, ce fonctionnement offre de nombreuses similitudes avec d'autres pays africains, questionnant l'idée d'un exceptionnalisme rwandais.

Depuis son avènement au pouvoir, le Front patriotique rwandais a fait de la lutte contre la corruption son fer de lance. Elle est aujourd'hui incontournable, dans la rue comme dans les bureaux de l'administration. Elle a acquis tant une fonction économique que sociale, dans le but d'atteindre les objectifs de développement, de doter le Rwanda d'une économie à revenu intermédiaire, sans tension ethnique et jouissant d'une gouvernance efficace ${ }^{1}$. En déployant une stratégie de «tolérance zéro vis-à-vis de la corruption ${ }^{2}$ », l'État a mis en place des moyens conséquents pour mettre fin à ces pratiques. Le Rwanda offre ainsi un terrain d'étude original pour la lutte anti-corruption, tant celleci a été érigée en modèle à suivre et utilisée comme image de marque dans le discours officiel rwandais. Mais derrière cet engagement formel, comment les parties prenantes s'approprient-elles cette politique au quotidien? Cette étude de cas se propose d'étudier l'évolution des pratiques de corruption 
entre 2013 et 2019 en examinant l'application de la politique d'ordre auprès des taxis-motos sur les routes de Kigali.

Cette étude de cas se rapproche d'autres recherches visant à saisir comment les réformes de l'État sont menées au Rwanda. Elles interrogent notamment le contraste entre le contrôle formel que le régime donne à voir et l'informalisation de l'État. Par exemple, Gaynor ${ }^{3}$ et Purdeková4 étudient le contrôle étatique de la population rwandaise à travers l'instrumentalisation de la participation civique, au nom de la bonne gouvernance ou du développement. De même, Baez Camargo montre les attentes multiples et conflictuelles qui se nouent entre les normes légales et les normes sociales de solidarité qui s'imposent aux individus ${ }^{5}$. Cependant, aucune recherche n'a jusqu'à aujourd'hui cherché à analyser les pratiques de petite corruption dans le transport routier au Rwanda et le détournement de la politique anti-corruption par les acteurs.

Les taxis-motos de Kigali constituent un microcosme particulièrement fécond pour éclairer la production et la perpétuation des pratiques de corruption. Il s'agit d'un groupe d'individus très exposé à la corruption, qui a ainsi développé un savoir-faire particulier en la matière. En outre, bien qu'il s'agisse à la base d'un secteur économique informel, les taxis-motos au Rwanda en sont venus à être très régulés ${ }^{6}$. Dans le but de surveiller leur conduite, ils sont regroupés au sein de coopératives depuis 20087, ces dernières maintenant des liens étroits avec la police routière. Le prisme de la corruption dans ce milieu particulier permet ainsi d'examiner l'assujettissement aux normes, formelles et informelles, et, réciproquement, le rôle que jouent celles-ci dans le maintien des pratiques collectives. L'enjeu est notamment d'analyser les facteurs ou les déclencheurs de la corruption afin d'appréhender le «fonctionnement informel généralisé de l'État ${ }^{8}$ ». La notion de corruption est ici entendue comme tout abus de pouvoir légitime à des fins de profits personnels, des pots-de-vin au favoritisme.

L'originalité de l'étude repose également sur son approche méthodologique qui met au cœur de la réflexion les interactions entre usagers et représentants

3. N. Gaynor, " "A Nation in a Hurry": The Costs of Local Governance Reforms in Rwanda », Review of African Political Economy, vol. 41, n S1, 2014, p. S49-S63.

4. A. Purdeková, «"Even if I Am not here, there Are so many Eyes”: Surveillance and State Reach in Rwanda", The Journal of Modern African Studies, vol. 49, n 3, 2011, p. 475-497.

5. C. Baez Camargo, Corruption, Social Norms and Behaviours: A Comparative Assessment of Rwanda, Tanzania and Uganda, Bâle, Basel Institute on Governance, 2017.

6. T. Goodfellow, "Taming the "Rogue" Sector: Studying State Effectiveness in Africa through Informal Transport Politics», Comparative Politics, vol. 47, n 2, 2015, p. 127-147.

7. Pour plus de détails sur la création des coopératives, voir W. Rollason, Motorbike People: Power and Politics on Rwandan Streets, Lanham, Lexington Books, 2019, p. 46.

8. G. Blundo et J.-P. Olivier de Sardan, Everyday Corruption and the State: Citizens and Public Officials in Africa, Londres, Zed Books, 2006. 
de l'autorité de l'État. À l'instar de Cissokho qui étudie la privatisation de l'État dans la gestion du transport routier au Sénégal ${ }^{9}$, l'analyse se concentre ici sur la cooptation d'acteurs en dehors de l'État (en l'occurrence ici les agents de sécurité des coopératives) pour l'implantation des politiques publiques afin de comprendre cette coproduction de l'ordre et l'enchâssement social des politiques publiques ${ }^{10}$. En situant l'enquête dans un temps suffisamment long, l'étude permet ainsi d'appréhender l'appropriation des politiques publiques par les agents, mais aussi l'évolution des multiples formes de légitimité de l'action des agents et des administrés (le pluralisme normatifi1). Pour ce faire, l'étude fait appel à l'approche des normes sociales développée par C. Bicchieri12, qui considère qu'une norme se perpétue en raison de la fréquence de certains comportements liés à elle (développant alors ce que Bicchieri appelle une attente empirique), de croyances en l'obéissance d'autrui à la norme (appelées ici attentes normatives) et en un système de sanctions et de récompenses au sein d'un groupe d'individus ${ }^{13}$.

Pour saisir les aspects normatifs et empiriques des pratiques de corruption, 120 entretiens avec des motards et des agents de sécurité ont été effectués en 2013 et en 2019. Les entretiens se sont déroulés dans des bars en kinyarwanda (avec un traducteur). Les interviewés étaient choisis de manière aléatoire, sur les parkings ou dans la rue, sur une base volontaire d'échange. Les deux tiers des entretiens ont été réalisés avec les taxis-motos, les agents de sécurité étant moins nombreux et surtout plus réticents à échanger. Les tentatives d'entretien avec les responsables des coopératives et les forces de l'ordre ont été peu fructueuses: les premiers se sont montrés peu loquaces et seuls deux policiers ont accepté un entretien. Les taxis-motos et les agents de sécurité, informés de l'utilisation et de l'anonymisation des données collectées, ont été principalement interrogés sur le fonctionnement des coopératives, leur rapport aux pratiques de corruption et leurs relations avec les autres acteurs du milieu. Des vignettes, présentant des situations particulières, ont également été établies afin de comprendre l'ancrage social de la corruption

9. S. Cissokho, «Être officiel ou faire officiel? Sur deux styles de barrages routiers en Afrique de l'Ouest (Ghana/Sénégal)», Critique internationale, n 83, 2019, p. 167-189.

10. T. Bierschenk et J.-P. Olivier de Sardan (dir.), States at Work: Dynamics of African Bureaucracies, Leiden/Boston, Brill, 2014.

11. G. Anders, Civil Servants in Malawi: Cultural Dualism, Moonlighting and Corruption in the Shadow of Good Governance, Rotterdam, Erasmus University Rotterdam, 2005.

12. C. Bicchieri, Norms in the Wild: How to Diagnose, Measure, and Change Social Norms, New York, Oxford University Press, 2016, p. 64-65.

13. Autrement dit, une pratique de corruption pourra être considérée comme une norme sociale si elle correspond 1) à une attente empirique (une conformité est attendue envers un comportement donné) ; 2) à une attente normative a) si une conformité envers une croyance collective est déterminante dans le comportement des acteurs; b) si la croyance en un système de sanction sociale influence les pratiques et les préférences des acteurs. 
et l'importance des normes de réciprocité au sein des réseaux de référence des taxis-motos.

Ces entretiens ont été complétés par des observations durant les heures d'affluence sur les parkings des taxis-motos, principalement celui de Nyabugogo, centre des transports routiers de Kigali. Les parkings sont des espaces à ciel ouvert, délimités le long de la route, où les taxis-motos sont obligés de stationner (et de payer) en attendant un client. D'autres observations ont eu lieu devant les coopératives de taxis-motos afin de mieux saisir le lien entre taxis-motos et coopératives. Des observations en catimini ont été réalisées en marge des check-points de la police afin d'observer les interactions entre les forces de l'ordre et les taxis-motos. Enfin, des observations participantes en tant que passager ont également été réalisées, permettant des discussions informelles avec les chauffeurs et l'observation des interactions entre taxismotos, agents de police et agents de sécurité.

L'ensemble de ce matériel est mobilisé pour comprendre comment les politiques publiques de maintien de l'ordre sur les routes de Kigali sont détournées et utilisées par les différents acteurs dans le milieu des taxismotos. Après avoir présenté l'environnement dans lequel évoluent les acteurs, l'étude présente ensuite les logiques sociales et économiques à l'œuvre dans le fonctionnement de l'État au Rwanda, puis les pratiques des acteurs et leur portée normative. Enfin, la conclusion discutera de l'apport de cette étude de cas à la compréhension du phénomène de la corruption, de l'État au Rwanda et, de manière plus générale, de l'État en Afrique.

\section{LE MILIEU DES TAXIS-MOTOS DE KIGALI}

\section{La vie de taxi-moto, entre le marteau et l'enclume}

Il y a plus de 15400 taxis-motos à Kigali ${ }^{14}$. Ils représentent un élément essentiel du système de transport de la ville ${ }^{15}$. Les chauffeurs sont à l'image de la capitale du Rwanda: jeunes, la moitié d'entre eux étant originaire de l'intérieur des terres et en recherche d'opportunités économiques. Les motards travaillent en moyenne 11 heures par jour. Leurs conditions de travail sont difficiles: fatigue, conduite dangereuse et densité du trafic routier sont autant de facteurs multipliant les risques d'accidents (qui sont assez fréquents).

14. Rwanda Utilities Regulatory Authority, Statistics in Transport Sector as of March of the Year 2018, Kigali, Rwanda Utilities Regulatory Authority, 2018.

15. W. Rollason, Youth Development Success amongst Motorcycle Taxi Drivers in Kigali, Londres, Brunel University, 2017. 
Les conditions de travail ont peu évolué entre 2013 et 2019. La plupart des motards disent avoir quitté leur province rurale avec leurs maigres économies après un cursus scolaire court en espérant trouver une vie meilleure à Kigali. Comme le montre l'extrait d'entretien ci-dessous, les motards choisissent ce travail en raison de ses gains rapides et de ses perspectives de carrière (l'ambition la plus répandue chez les motards étant de devenir chauffeur de taxi-voiture):

\section{Extrait d'entretien avec Alexis, taxi-moto ${ }^{16}$}

Moi : Pouvez-vous vous présenter?

Alexis: Je m'appelle Alexis, je suis taxi-moto depuis 3 ans. Je suis originaire de Nyanza où j'étais coiffeur. J'ai pensé que je pouvais gagner plus comme motard alors je suis venu à Kigali. Ma femme m'a suivi. J'ai toujours voulu faire de la moto, c'est une passion. Moi : Qu'est-ce que vous aimez dans la vie?

Alexis: Ce que j'aime le plus dans la vie, c'est la paix, la tranquillité, c'est-à-dire pouvoir travailler librement. J'ai ma propre moto et toutes les autorisations mais je ne me sens pas libre car il y a toujours des gens qui me demandent de l'argent. Ce sont les policiers et les agents de sécurité. Je n'aime pas le métier à cause de ces obstacles.

Moi: Qu'est-ce que cela représente pour vous?

Alexis: Cela représente beaucoup d'argent. Les gens nous accusent de crimes qu'on n'a pas commis. Par exemple, quelqu'un vient et dit: «Tu n'es pas garé correctement. » À ce moment-là, on accepte de payer le montant qu'il demande ou bien on négocie.

Comme le montre l'extrait ci-dessus, la thématique de la corruption est arrivée très vite dans l'entretien sans que mes interlocuteurs sachent que je travaillais sur ce sujet et sans que la question soit explicitement formulée. En 2013 comme en 2019, cette thématique était récurrente lors de mes entretiens. Ainsi, sans fard, les taxis-motos interrogés affirment «mes difficultés c'est la police qui nous arrête et nous demande la corruption ${ }^{17}$ », ou encore: «Nous sommes oppressés dans notre travail. Le plus gros problème pour nous, ce sont les agents de sécurité et les coopératives qui nous volent notre $\operatorname{argent}^{18}$ !» Il y a cependant une évolution de ces pratiques. En 2013, environ $85 \%$ des 70 motards interrogés déclaraient avoir payé au moins un pot-devin à la police dans le mois précédant l'enquête. En 2019, seulement $25 \%$ des 30 motards interrogés affirment la même chose, contre $100 \%$ affirmant avoir

16. Entretien avec un taxi-moto, Nyabugogo, 9 septembre 2013.

17. Ibid.

18. Entretien avec un taxi-moto, Nyamirambo, 26 septembre 2019. 
corrompu des agents de sécurité. Ces derniers sont devenus le principal problème mis en exergue par les taxis-motos, en lien avec un sentiment d'entrave de leurs activités, mais aussi d'accroissement du coût de la corruption.

Le chiffre d'affaires mensuel moyen d'un taxi-moto en 2019 est d'environ 250000 francs rwandais, celui-ci ayant peu évolué depuis 2012. Comme les taxis-motos ne sont pas, pour la plupart, propriétaires de leur véhicule, ils doivent payer 125000 francs rwandais par mois à leur «patron » pour utiliser le véhicule. Si l'assurance et l'entretien de la moto sont à la charge du propriétaire, l'essence (75000 francs rwandais par mois), le parking (7500 francs rwandais par mois) et les taxes (7500 francs rwandais par mois) doivent être assumés par les motards. Malgré tous ces frais, leur rémunération est supérieure au salaire moyen rwandais; selon les entretiens avec les motards, ces derniers peuvent épargner chaque mois jusqu'à 50000 francs rwandais. Cette somme, qui dépasse le salaire des policiers et des agents de sécurité, peut générer des envies: «Les policiers et les agents de sécurité nous demandent toujours de l'argent, nous sommes des vaches à lait ${ }^{19}$ ! \ Ces bénéfices sont donc calculés sans compter les amendes et la corruption qui ont un impact direct sur les gains des motards et qui sont intégrées de force dans leur budget de fonctionnement.

Les motards forment un groupe disparate, mais unifié. Outre leur uniformité vestimentaire (à travers leur gilet officiel), ils affichent des liens de solidarité, ainsi que le sentiment de difficultés partagées, pour ce qui est leur travail, mais aussi de leur cadre de vie, éloigné de leurs familles restées à la campagne. En outre, la communauté des motards est institutionnalisée par un enregistrement obligatoire auprès d'une coopérative. L'ensemble de ces facteurs poussent les motards à considérer leurs pairs comme leur réseau de référence, influençant leurs attentes individuelles et formant un cadre de compréhension des normes propres à leur milieu. Comme le montrent Ibrahim et Bize au Kenya ${ }^{20}$, les moments de socialisation des taxis-motos sont un vecteur de structuration de l'environnement professionnel qui facilite dans le même temps la reproduction des pratiques de corruption.

En effet, les normes de conduite se forment et se perpétuent lors des échanges entre taxis-motos, en particulier sur les parkings. Lorsque les motards se garent à la station Nyabugogo, les conversations évoquent généralement les accidents, les problèmes avec les clients, mais aussi et surtout les rencontres avec les agents de sécurité et la police. Ces échanges font partie des processus de normalisation de la corruption, une compréhension et une appréhension 
des interactions avec les agents de police et de sécurité. Les motards échangent conseils et points de vue sur la corruption, banalisée par des blagues ou des anecdotes sur leurs propres expériences en la matière. Dans l'extrait qui suit, est ainsi narrée une histoire racontée sur le ton de la blague par un taxi-moto à son collègue, après que ce dernier venait de "payer la corruption» à un check-point policier:

\footnotetext{
«Un motard est arrêté et sa moto embarquée. Le motard s'arrange pour connaître l'adresse du policier et passe la nuit devant sa maison. À 3 h00 du matin, le policier sort de chez lui pour uriner et tombe sur le motard. Le policier pense appeler au secours, mais le motard lui dit qu'il ne veut pas lui faire de mal, il veut juste savoir comment sauver son gagnepain pour ses enfants et c'est pour ça qu'il l'avait suivi jusque chez lui. Étonné, le policier lui dit de rentrer et de passer le voir au bureau pour récupérer sa moto. Le motard passe récupérer la moto et, le soir, il va chercher le policier pour lui payer à boire et finalement ils deviennent bons amis ${ }^{21}$ !»
}

Si une telle situation (attendre des heures, et de nuit, devant une maison) paraît en elle-même difficile à croire, l'histoire n'en met pas moins en exergue une certaine témérité des taxis-motos mais aussi et surtout un côté pragmatique: il s'agit d'entretenir des relations amicales avec les policiers pour «faciliter le travail». Cette socialisation professionnelle permet aux motards de réduire les risques et l'incertitude lorsqu'ils sont confrontés à des situations similaires. Soulignons cependant qu'il s'agit d'une influence normative a minima car il n'y a pas de sanction sociale si un taxi-moto dévie de la norme en refusant de se corrompre ou en adoptant d'autres attitudes vis-à-vis des agents. Par rapport à leur réseau professionnel de référence, les motards sont indépendants et développent des attentes personnelles et non collectives. En effet, comme le souligne bien l'anecdote ci-dessus, sur leur lieu de travail et notamment face aux agents de police et de sécurité, les motards sont seuls, malgré leur ancrage auprès des coopératives.

\section{Les taxis-motos solitaires les uns des autres}

Regroupés au sein de l'Association des taxis-motos du Rwanda (Assetamorwa) depuis la fin des années 1990, les motards avaient alors mauvaise réputation. Associé aux attaques attribuées aux Forces démocratiques pour la libération du Rwanda (FDLR) et considéré comme un regroupement de délinquants, le milieu des taxis-motos était alors dans le collimateur des autorités. Afin d'assurer plus de sécurité et de mieux contrôler le secteur, des coopératives 
de taxis-motos furent créées en 2007 suite à la loi sur les coopératives ${ }^{22}$, puis rassemblées en 2008 sous l'autorité de la Fédération des coopératives des conducteurs des taxi motos (Ferwacotamo).

Ces coopératives ont la responsabilité d'assurer la sécurité du secteur: identification et enregistrement de tous les chauffeurs de taxis-motos et délivrance d'un gilet numéroté pour chaque motard en fonction de sa coopérative, de son secteur et du parking auquel il est rattaché. Leur enregistrement auprès des coopératives est obligatoire pour obtenir une licence et avoir le droit d'exercer. Au regard de leur activité et de la loi, cette gestion du secteur des transports s'apparente à une concession informelle de service public. Dans la pratique, les coopératives offrent également de multiples services comme la facilitation d'obtention de crédit bancaire, l'achat de motos, la gestion des conflits entre les motards et les propriétaires de véhicules, etc. Il y a aujourd'hui 147 coopératives de taxis-motos à travers le pays ${ }^{23}$; à Kigali, certaines d'entre elles, comme Tubane Hafi, regroupent jusqu'à 800 motards ${ }^{24}$.

Ces coopératives sont des ensembles très structurés, avec une assemblée générale, une direction et un comité d'inspection. Des chefs d'équipes sur les parkings surveillent la conduite des taxis-motos et informent les agents de sécurité, lesquels doivent eux-mêmes rendre compte auprès des agents de sécurité au niveau du district, qui eux-mêmes répondent aux agents de sécurité au niveau de la ville, le tout sous l'autorité de la Ferwacotamo. Ainsi, il s'agit d'un système de surveillance hiérarchisé et vertical calqué sur la division formelle du territoire, en phase avec le système de contrôle et de discipline étatique ${ }^{25}$.

Ces agents de sécurité sont des anciens taxis-motos embauchés par les coopératives. L'embauche est très souvent corrélée à une période sans moto (accident, vol, panne, etc.). De bonnes relations sont également essentielles pour obtenir le poste, généralement attribué par cooptation, sans exiger de compétence particulière:

«C'est le président de ma coopérative qui est venu m’approcher et me proposer de devenir agent de sécurité parce qu'il voyait que je venais de passer quelques mois sans moto à conduire. Moi, j'étais très connu pour être un type jovial qui s'entend bien avec tout le monde, c'est pourquoi j'ai été choisi comme agent de sécurité26.»

22. Republic of Rwanda, Law No 50/2007 of 18/09/2007 determining the establishment, organisation and functioning of cooperative organisations in Rwanda.

23. Rwanda Utilities Regulatory Authority, Statistics in Transport Sector..., op. cit.

24. Ibid.

25. A. Purdeková, " "Even if I Am not here, there Are so many Eyes"...», art. cité.

26. Entretien avec un agent de sécurité, Kicukiro, 6 juillet 2019. 
Pour les taxis-motos, «les agents de sécurité, ce sont des taxis-motos qui n'ont pas réussi ${ }^{27}$ ».

Les coopératives collectent mensuellement une cotisation auprès de leurs membres (5000 francs rwandais), ainsi que des droits annuels d'adhésion (environ 60000 francs rwandais), servant officiellement à financer leurs frais de fonctionnement, la Ferwacotamo ainsi qu'une caisse de prévoyance et d'investissement des coopératives. Cependant, des allégations de détournements de fonds, d'extorsion ${ }^{28}$ et de manque de coordination ${ }^{29}$ ont entaché la réputation des coopératives. Tous les motards interviewés les désavouent: «Ils n'ont que l'argent comme langage, ce sont des voleurs ${ }^{30}$ !» En 2017, une pétition des motards évoquant le manque de redevabilité et la corruption au sein des coopératives est portée à l'attention du parlement rwandais ${ }^{31}$. L'année suivante, l'Agence rwandaise des coopératives parle d'un détournement de 205 millions de francs rwandais ${ }^{32}$.

En 2018, suite aux «différents problèmes occasionnant le mécontentement des membres eu égard aux agissements des agents chargés de la sécurité au sein desdites coopératives ${ }^{33}$ ", l'Agence rwandaise des coopératives requit le changement de statut des agents de sécurité qui sont devenus des «agents de discipline », cette notion étant plus restreinte et moins équivoque que celle de sécurité. Cependant, personne n'utilise cette appellation, ce qui témoigne du flou entretenu autour de leur statut, mais aussi de l'écart entre les régulations et les pratiques. En 2019, les instructions de l'agence vont même jusqu'à établir des directives pour «les punitions des organes de sécurité» afin de mieux définir les prérogatives des agents et les sanctions en cas de non-respect des consignes ${ }^{34}$. Formellement, «l'agent chargé de la sécurité n'est pas autorisé à taxer d'amende les membres de la coopérative ni à les percevoir ${ }^{35}{ }_{\text {». }}$

27. Entretien avec un taxi-moto, Nyabugogo, 12 octobre 2013.

28. E. Ntirenganya, «Coops Agency Moves to Streamline Taxi-Moto Operators' Cooperatives», The New Times, 8 juin 2018.

29. N. Bishumba, «RCA Moves to Fix Challenges Raised by Taxi Moto Operators», The New Times, 21 décembre 2018.

30. Entretien avec un taxi-moto, Gasabo district, 12 septembre 2013.

31. R. Rwirahira, «Taxi-Moto Operators Petition Parliament over Mismanagement of their Organization", The New Times, 30 mars 2017.

32. C. Amani, «RCA montre des changements après la réforme des coopératives des motards", Imvaho Nshya, 18 septembre 2018.

33. Agence rwandaise des coopératives, Instructions $n^{\circ} 0211 / 2018$ du 3 avril 2018 régissant le personnel chargé de la sécurité (disciplinary officers) au sein des coopératives des motards au Rwanda, Kigali, Agence rwandaise des coopératives, 3 mai 2018.

34. Agence rwandaise des coopératives, Instructions $n^{\circ} 002 / 2019$ du 15 avril 2019 régissant le personnel chargé de la sécurité au sein des coopératives des transporteurs sur motocyclettes au Rwanda, Kigali, Agence rwandaise des coopératives, 15 mai 2019.

35. Ibid. 
Malgré ces changements, les taxis-motos ne se sentent pas moins démunis au sein de leurs coopératives. Certains expliquent que «les dirigeants [...] ne peuvent pas contredire l'État ${ }^{36}$ », d'autres que «les assemblées générales sont dominées par ceux qui ne sont pas taxis-motos ${ }^{37}$ », d'autres encore que «les anciens militaires sont ceux qui choisissent qui va diriger les associations ${ }^{38}$ ». Cela corrobore le sentiment d'isolement des motards face à cette imbrication de multiples formes d'autorité, formelles et informelles ${ }^{39}$.

Comme l'attestent les entretiens, les coopératives représentent une solidarité a minima pour les taxis-motos. Pour comprendre l'importance de leur réseau de référence, durant les entretiens, les motards étaient confrontés à une vignette dans laquelle leur collègue taxi-moto demandait leur aide dans une affaire de corruption d'un agent de la police. Les motards se montrent tous peu enthousiastes à l'idée de prendre des risques pour leur réseau professionnel sauf s'ils peuvent en retirer un gain personnel:

«Les paroles seules ne suffisent pas pour demander du paturage pour une vache ${ }^{40}$; personne ne fait plus des choses sans qu'il pense qu'il pourra y gagner quelque chose et, pour les motards, c'est pire que dans les autres métiers ${ }^{41}$.»

Lorsque le collègue était remplacé par un proche dans les vignettes, les résultats montraient au contraire une forte obligation de solidarité:

\section{Extrait d'un entretien avec un taxi-moto ${ }^{42}$}

Joshua est taxi-moto à Kigali. Un policier l'arrête. Ce jour-là, Joshua a oublié son casque et il n'a pas tous ses papiers en règle; sa moto est saisie. Joshua comprend que s'il souhaite récupérer sa moto, il devra payer une somme importante, environ 60000 francs rwandais. II pense faire appel à son cousin policier, Jean-Paul, pour jouer le rôle d'intermédiaire et négocier son cas.

Moi : Qu'est-ce qu'un motard ferait dans sa situation?

36. Entretien avec un taxi-moto, Kicukiro, 10 septembre 2013.

37. Entretien avec un taxi-moto, Kiyovu, 8 septembre 2019.

38. Entretien avec un taxi-moto, Nyarutarama, 10 décembre 2019.

39. Comme l'explique Ingelaere, «les structures horizontales de cooptation amplifient considérablement les positions "d'autorité" dans la société rwandaise et renforcent la chaîne de responsabilité envers le centre». Voir B. Ingelaere, «What's on a Peasant's Mind? Experiencing RPF State Reach and Overreach in Post-Genocide Rwanda (2000-10) », Journal of Eastern African Studies, vol. $8, \mathrm{n}^{\circ} 2,2014$, p. 224.

40. Traduction du kinyarwanda de «Amatama masa ntasabira inka igisigati».

41. Entretien avec un taxi-moto, Gasabo district, 10 décembre 2019.

42. Entretien avec un taxi-moto, Nyabugogo, 8 décembre 2019. 
Damien: Le motard chercherait à obtenir clémence ou à payer la corruption. Si son cousin est fort dans la police, le motard penserait qu'il va résoudre son problème.

Moi: Qu'est-ce que les amis et la famille de Joshua attendent de lui dans cette situation? Damien: Les amis et la famille vont avoir peur que ça ne marche pas et que Joshua soit puni.

Moi : Qui a le plus d'influence sur la décision de Joshua? Les motards, sa famille?

Damien: C'est Jean-Paul qui va jouer un grand rôle dans cette situation et même dans toutes les décisions que Joshua va prendre. Les gens de la famille vont chercher l'argent pour aider. Les motards s'en moquent complètement. Il y a beaucoup de motos saisies et les motards ne savent même pas à qui elles appartiennent.

Si cela corrobore le rôle de l'entraide familiale dans le maintien de certaines pratiques de corruption ${ }^{43}$, cela souligne par contraste la faiblesse des obligations des motards envers leurs collègues. L'individualisme et la méfiance entre motards semblent d'ailleurs plus développés en 2019 qu'en 2013. Les motards évoquent des pratiques de délation entre taxis-motos lorsque certains d'entre eux risquent de nuire à la profession ou lorsque des «espions» travaillant à la solde du gouvernement dénoncent les méfaits de certains taxis-motos. Comme le souligne Ansoms et Cioffo, à travers l'utilisation de moyens informels, tel que des infiltrés au sein d'une communauté, l'État est présent à tous les niveaux de la sociétét ${ }^{4}$.

En outre, une collaboration formelle est établie entre la police routière et les coopératives de motards, dont les agents de sécurité sont la pierre angulaire. Les directives de l'Agence rwandaise des coopératives stipulent que «les agents [de sécurité] peuvent aller travailler n'importe où sur demande de la fédération ou de la direction de la police routière ${ }^{45} »$. De plus, les agents peuvent être mobilisés «en cas d'une opération organisée par la police et qui en fait la demande à la coopérative pour mettre à disposition ses agents et aider dans cette opération ${ }^{46}{ }$. Les agents de sécurité sont donc considérés comme des auxiliaires pour le maintien de l'ordre sur la voie publique. Comme le souligne l'un d'entre eux, «en tant qu'agent de sécurité, mes attributions me viennent de la police. Parfois, nous recevons un ordre spécifique, un avis

43. C. Baez Camargo et L. Koechlin, «Informal Governance: Comparative Perspectives on Co-Optation, Control and Camouflage in Rwanda, Tanzania and Uganda», International Development Policy/Revue internationale de politique de développement, $\mathrm{n}^{\circ} 10,2018, \mathrm{p} .78-100$.

44. A. Ansoms et G. D. Cioffo, «The Exemplary Citizen on the Exemplary Hill: The Production of Political Subjects in Contemporary Rural Rwanda», Development and Change, vol. 47, $\mathrm{n}^{\circ}$ 6, 2016, p. $1247-1268$.

45. Agence rwandaise des coopératives, Instructions $n^{\circ}$ 002/2019 du 15 avril 2019..., op. cit. 46. Ibid. 
de recherche, et nous devons trouver le fautif ${ }^{47}$ ». Cette collaboration est ici importante car elle explique notamment le glissement potentiel de l'autorité des agents de police entre les mains des agents de sécurité.

\section{L'ENCHÂSSEMENT SOCIAL DES POLITIQUES PUBLIQUES}

\section{La répression de la corruption}

La promotion de l'intégrité publique et privée au Rwanda s'est appuyée sur des moyens importants, tant juridiques et disciplinaires que socio-culturels. Sans volonté d'exhaustivité, depuis 1999 ont vu le jour des programmes de rééducation des populations (Itorero et Ingando) pour promouvoir l'intégrité et le civisme ${ }^{48}$, une unité anti-corruption au sein de l'Office rwandais des recettes, de nombreuses campagnes de sensibilisation à travers tout le pays et un strict encadrement légal des conduites au sein des secteurs privés et publics. Citons notamment l'adoption en 2003 d'une loi anti-corruption ${ }^{49}$ (mise à jour en $2018^{50}$ ) punissant de 2 à 10 ans d'emprisonnement et d'une amende allant jusqu'à dix fois la valeur du profit illicite toute personne impliquée directement ou indirectement dans une affaire de corruption. Mais c'est surtout la stratégie nationale de lutte contre la corruption adoptée dès 201251, avec le mot d'ordre d'une "tolérance zéro vis-à-vis de la corruption », qui marqua le début d'une réforme de l'État et d'une rationalisation de son action à des fins affichées d'intégrité.

$\mathrm{Au}$ sein de la police, des réformes institutionnelles furent entreprises: un département d'inspection des services fut créé 52 , ainsi qu'une unité disciplinaire ${ }^{53}$ et un centre d'éthique pour promouvoir des standards professionnels ${ }^{54}$. Aujourd'hui, toute nouvelle recrue reçoit des briefings éthiques de la part des officiers seniors, un entraînement sur les normes éthiques et des cours sur le code de conduite à tenir. Des procédures d'évaluation furent également mises en place, ainsi qu'une ligne verte pour

47. Entretien avec un agent de sécurité, Kicukiro, 8 septembre 2013.

48. A. Purdeková, " "Even if I Am not here, there Are so many Eyes"...», art. cité.

49. Republic of Rwanda, Law $n^{\circ} 23 / 2003$ of 07/08/2003.

50. République du Rwanda, Loi $n^{\circ}$ 54/2018 du 13/08/2018 relative à la lutte contre la corruption, 2018.

51. Republic of Rwanda, Rwanda Anti-Corruption Policy, op. cit.

52. Rwanda National Police, «Transparency International Chairperson Visits Rwanda National

Police», 17 février 2014.

53. «Police Tackles Insider Crime», The Independent, 9 août 2013.

54. «RNP Ethic Center to Instill Integrity», Hope Magazine, 5 mai 2012. 
dénoncer toute forme de corruption ${ }^{55}$. Une simple suspicion de corruption peut aujourd'hui entraîner un renvoi, un éloignement des suspects de leurs responsabilités, ainsi qu'une "rééducation» dans une unité disciplinaire ${ }^{56}$. Entre 2010 et 2017, plus de 600 officiers furent licenciés pour avoir sollicité ou accepté un pot-de-vin. De plus, de nombreux agents de police, agents de sécurité et taxis-motos ont été condamnés pour corruption, comme le montre la base de données de l'Ombudsman ${ }^{57}$. Ce dernier publie annuellement le nom des condamnés et de leurs parents, cette mise au pilori pouvant avoir pour effet de stigmatiser les familles ${ }^{58}$. Soulignons également que tous les condamnés le sont sur base d'acceptation ou de versement de pot-de-vin, ce qui correspond à une conception purement économique et $a$ minima de la notion de corruption.

D'autres mesures de prévention furent également mises en œuvre pour détourner les agents de police de la corruption, tels que l'amélioration de la sécurité sociale, la rotation des employés ${ }^{59}$, mais aussi et surtout le doublement des salaires des officiers de police en $2016^{60}$. En effet, les bas salaires étaient souvent considérés comme une des raisons principales de corruption des policiers de la sécurité routière ${ }^{61}$, ces derniers étant auparavant, selon Baker, dans la logique d'une économie de survie ${ }^{62}$. Le salaire brut moyen mensuel d'un officier était de 70799 francs rwandais ${ }^{63}$ avant cette augmentation ${ }^{64}$, soit un salaire équivalent au revenu brut moyen par habitant (72000 francs rwandais en 2016) ${ }^{65}$.

Quant aux agents de sécurité des coopératives, leur formation succincte n'aborde pas la corruption. Ils sont payés 60000 francs rwandais par mois, une somme qu'ils estiment souvent insuffisante. Ainsi que l'exprime l'un

55. Rwanda National Police, «A Glance at the Rwanda National Police Response to Graft», 13 avril 2015.

56. Republic of Rwanda, Art 13 (2), Ministerial instructions $n^{\circ}$ 003/12 of 17/9/2012 establishing police code of conduct, 2012.

57. Republic of Rwanda, Ombudsman, Corruption Convicts Database 2013, 2014, 2015.

58. E. Ntirenganya, «Should Names of Convicts' Parents Be Published?», The New Times, 17 août 2020 .

59. «Police Vow to Slash Corruption to Zero Scale», Hope Magazine, 10 septembre 2012.

60. «Kagame's Encounter with Corruption Could Have Led to Doubling Police Salaries in 2016», The Chronicles, 12 juin 2019.

61. A. Bozzini, «Fighting Corruption while Undermining Accountability: The Case of Rwanda», Working Package 3, Hambourg, German Institute of Global and Area Studies, 2014.

62. B. Baker, «Reconstructing a Policing System out of the Ashes: Rwanda's Solution», Policing and Society, vol. $17, \mathrm{n}^{\circ} 4,2007$, p. 344-366.

63. Un euro équivaut à environ 1000 francs rwandais, le taux de change étant équivalent entre 2013 et 2019.

64. Republic of Rwanda, Salary Scale for Police Officers, Kigali, Republic of Rwanda, 2012.

65. Banque mondiale, Rwanda, 2016, <https://donnees.banquemondiale.org/pays/rwanda>, consulté le 19 novembre 2020. 
d'entre eux: «Selon le salaire que je gagne à la fin du mois, il est presque impossible de ne pas être tenté par la corruption, [...] et nous n'avons aucune couverture sociale ni d'assurance médicale ${ }^{66}$.» Soulignons également le cadre administratif flou dans lequel évoluent les agents de sécurité: ces derniers signent un contrat de travail avec leur coopérative mais agissent selon les directives de la Ferwacotamo. Les agents de sécurité disposent donc d'une marge de manœuvre importante, puisqu'ils sont peu redevables envers leur mandataire et agissent pour une entité qui ne rend pas compte de leurs activités, les mesures mises en place par la police pour diminuer la corruption ne s'appliquant de surcroît pas à eux.

\section{La gestion déléguée de la sécurité routière}

L'augmentation exponentielle du nombre de véhicules en circulation au Rwanda (de 61000 véhicules enregistrés en $2009^{67}$ à 180137 en 201868) peut expliquer la diminution des barrières de la police routière sur les routes. Il est également possible qu'en raison de la forte répression contre la corruption et du nombre impressionnant de policiers licenciés, les services de police décidèrent de limiter les interactions entre leurs agents et le public. Quoi qu'il en soit, c'est en tout cas le retrait partiel des forces de police des voies publiques de Kigali qui a amené les agents de sécurité à s'arroger de facto une autorité de régulation de la conduite des taxis-motos, au nom de la sécurité routière. De fait, puisqu'il n'y a pas de délégation formelle et donc aucun encadrement de cette délégation, les directives évoquées plus haut mentionnant uniquement la mise à disposition des agents de sécurité lors d'opérations de la police, ces agents de sécurité s'arrogent également le droit d'arrêter les taxis-motos sur la voie publique alors que les textes directeurs ne stipulent qu'une responsabilité pour le maintien de l'ordre sur les parkings ${ }^{69}$.

Pour reprendre la formulation d'Olivier deSardan, il s'agit d'une "privatisation informelle» des normes de sécurité, qui apparaît à la fois «transgressive» et "palliative», dans le sens où «elle nuit gravement à la délivrance de services publics de qualité», mais dans le même temps «facilite de façon "bricolée" la

66. Entretien avec un agent de sécurité, Kicukiro, 9 décembre 2019.

67. World Health Organization, Global Status Report on Road Safety: Time for Action, Genève, World Health Organization, 2009.

68. World Health Organization, Global Status Report on Road Safety 2018, Genève, World Health Organization, 2018.

69. Agence rwandaise des coopératives, Instructions $n^{\circ} 0211 / 2018$ du 3 avril 2018..., op. cit. 
délivrance d'un service ${ }^{70}$ ». À travers leur méthode d'intervention, à savoir des patrouilles pouvant arrêter arbitrairement les motards n'importe où sur les routes, les agents de sécurité reproduisent une verticalité du pouvoir et réaffirment l'omniprésence de l'État tout en défendant leurs propres intérêts. On retrouve ici les ajustements situationnels et la compétition entre des formes d'institutionnalisation formelle et informelle des pratiques évoqués par Lund 71 .

Toutefois, les routes de Kigali ne sont pas des espaces de non-droit: la «privatisation informelle» des normes de sécurité par les agents fait aussi écho à une capacité d'autorégulation stratégique des acteurs afin de ne pas perdre leur statut et éviter des sanctions. D'un côté, les agents de sécurité sont redevables envers leur hiérarchie car ils ont obtenu leur poste (et que surtout ils peuvent le garder) au gré de leurs relations au sein de leur coopérative et/ou de la Ferwacotamo, ce qui renforce les pratiques de corruption: «Étant donné qu'on nous demande de l'argent pour garder notre travail, c'est sûr que nous aussi nous devons nous arranger pour en obtenir auprès des motards » affirme un agent de sécurité72. De même, Rollason évoque «un ensemble de relations sociales articulées autour d'un calcul des "avantages" » dans le maintien des pratiques de corruption au sein des coopératives ${ }^{73}$. D'un autre côté, la peur des infiltrés, des délations possibles, des représailles sont autant d'incitations à respecter les normes empiriques, sans les outrepasser. On constate ainsi une imbrication de la rationalité économique des acteurs dans «les contraintes et les dépendances liées à leurs relations interpersonnelles», pour reprendre la vision de Granovetter de l'enchâssement social ${ }^{74}$.

Les représentations sociales des comportements attendus doivent également être prises en compte dans l'analyse. En effet, l'utilisation d'un langage crypté et d'intermédiaires, les logiques de redistribution des gains par les agents sont autant d'exemples d'insertion des règles dans le contexte social. De même, le transfert d'autorité des policiers aux agents de sécurité illustre cette «institutionnalisation » du marché de la corruption, puisque les pratiques sont restées identiques malgré le changement d'acteurs. Ainsi, l'action de contrôle de l'État est imbriquée aux règles sociales propres au milieu professionnel

\footnotetext{
70. J.-P. Olivier de Sardan, La routine des comportements non-observants au sein des services publics nigériens. "Connaître la culture bureaucratique pour la réformer de l'intérieur». Rapport réalisé pour le Haut-Commissariat à la modernisation de l'État, Niamey, Lasdel, 2014.

71. C. Lund, "Twilight Institutions: Public Authority and Local Politics in Africa», Development and Change, vol. 37, $\mathrm{n}^{\circ} 4,2006$, p. 699.

72. Entretien avec un agent de sécurité, Kicukiro, 10 décembre 2019.

73. W. Rollason, Motorbike People..., op. cit.

74. M. Granovetter, «Economic Action and Social Structure: The Problem of Embeddedness", American Journal of Sociology, vol. 91, n³, 1985, p. 481-510.
} 
étudié et au contexte social élargi. En outre, en déléguant l'autorité aux agents de sécurité dans un cadre administratif opaque et facilitant les abus d'autorité, la gouvernance formelle a elle-même généré des voies de contournement du cadre coercitif, mêlant le formel à l'informel. En ce sens, cette conception de l'enchâssement social rappelle celle de Polanyi, où l'offre et la demande de corruption vont au-delà des relations interpersonnelles des acteurs et sont socialement organisées ${ }^{75}$.

\section{LA FORMALISATION DES JEUX DE POUVOIR}

Dans la définition de la sécurité sur les routes comme problème public, les taxis-motos apparaissent toujours comme une source de dangerosité. Comme évoqué plus haut, ils pâtissent encore d'une réputation de délinquants malgré le cadre très structuré dans lequel ils évoluent aujourd'hui. C'est notamment sur cette perception que les agents de sécurité basent leur légitimité d'action pour «corriger» leurs collègues et justifier leurs propres pratiques. Les taxismotos deviennent ainsi des vaches à lait en raison notamment de l'image véhiculée par les médias et les autorités, celle d'une jeunesse peu disciplinée, d'une communauté accueillant des fripouilles au sein de ses rangs ${ }^{76}$ et étant à l'origine de nombreux accidents de la route. Ils se retrouvent en porte-à-faux par rapport à l'image de marque de la ville et aux plans de développement centralisés (notamment le master plan de la ville de Kigali ${ }^{77}$ ). Les injonctions pour moderniser le secteur sont d'ailleurs nombreuses (imposition de compteurs, de paiement uniquement par téléphone, le développement de motos électriques, etc.) pour donner à voir une hypermodernité qui cache les pratiques «bricolées» évoquées ici.

Comme l'explique Rollason, les taxis-motos ne sont pas considérés par les autorités comme des acteurs politiques mais plutôt comme des perturbateurs de l'ordre public. L'utilisation politique de la communauté urbaine des taxismotos, qui est par exemple le propre de l'Ouganda voisin ${ }^{78}$, n'est pas de mise au Rwanda. En l'absence de compétition électorale ${ }^{79}$, ils ne sont pas un recours

75. K. Polanyi, La grande transformation. Aux origines politiques et économiques de notre temps, Paris, Gallimard, 1983.

76. «Taxi-Moto Operators Tipped on Crime Prevention», The New Times, 24 avril 2015.

77. W. Rollason, «Youth, Presence and Agency: The Case of Kigali's Motari», Journal of Youth Studies, vol. 20, n 10, 2017, p. 1277-1294.

78. T. Goodfellow et K. Titeca, «Presidential Intervention and the Changing "Politics of Survival" in Kampala's Informal Economy ", Cities, vol. 29, n 4, 2012, p. 264-270.

79. Bertelsmann Transformation Index, Rwanda, 2020, <https://www.bti-project.org/en/reports/ country-dashboard-RWA.html $>$, consulté le 19 novembre 2020. 
pour une mobilisation politique et sont donc plus fragiles. Dans un pays où le silence est institutionnalisée ${ }^{80}$, leurs résistances, par exemple à travers des manifestations à Kigali pour défendre leur secteur d'activité81, soulignent à la fois la force de cette communauté et la pression qu'elle doit affronter. L'existence même des coopératives et de la Ferwacotamo sont autant de moyens utilisés pour contrôler et «mater» les taxis-motos.

C'est parce qu'ils agissent au nom de l'État que les agents de sécurité se sentent forts: «Mes forces viennent des dirigeants de l'association et des institutions étatiques comme la Rwanda Cooperative Authority, la police et la ville de Kigali ${ }^{82}$.» L'enchâssement social des politiques d'ordre et de lutte contre la corruption souligne ainsi que le contrôle de l'État rwandais s'appuie à la fois sur des moyens formels et informels. Dans les rapports de force, les agents exploitent les règles formelles, le pouvoir symbolique des autorités, mais aussi la perception négative des taxis-motos et leur faible capacité de mobilisation.

Fort de cette légitimité pour les «corriger » et grâce à la complicité de leur réseau professionnel, les agents de sécurité négocient les règles de sécurité comme un répertoire d'actions possibles, à leur avantage. Si la corruption est censée être aussi dans l'intérêt des motards, elle n'en reste pas moins une spoliation à leurs yeux, ce qui génère parfois des tensions auxquelles les agents doivent faire face. «Nous leur demandons les papiers qu'ils n'ont pas et ils nous fuient, nous insultent, il y en a même qui nous frappent ${ }^{83}$.» Ce recours à la force montre à quel point les taxis-motos se sentent démunis face à ce qu'ils perçoivent comme une alliance entre les agents de sécurité et les forces de police. «Les agents de police routière ne nous écoutent pas, quand un motard commence à expliquer qu'il est innocent, que l'agent de sécurité le persécute, l'agent de police ajoute une autre faute qu'on appelle refus d'obtempérer ${ }^{84}$.»

Ces rapports de force montrent enfin que ce qui apparaissait à première vue comme un simple pot-de-vin est finalement aussi un abus de pouvoir. Cela souligne l'institutionnalisation de la corruption, dès lors que de telles pratiques sont soutenues aussi bien par des aspects structurels (la délégation d'autorité par la police routière, le manque de contrôle des agents de sécurité), des relations interpersonnelles et des rapports de pouvoir (la domination des taxis-motos par les agents de police ou de sécurité, la redistribution des

80. T. Goodfellow, "The Institutionalisation of "Noise" and "Silence" in Urban Politics: Riots and Compliance in Uganda and Rwanda» Oxford Development Studies, vol. 41, n 4, 2013, p 436-454.

81. W. Rollason, Motorbike People..., op. cit., p. 43.

82. Entretien avec un agent de sécurité, Kicukiro, 26 septembre 2019.

83. Entretien avec un agent de sécurité, Niboye, 15 septembre 2019.

84. Entretien avec un taxi-moto, Munanira 2, 23 septembre 2019. 
pots-de-vin par les agents à leur supérieur hiérarchique) que par des aspects normatifs (les attentes des agents et les sanctions à l'encontre des taxis-motos en cas de non-conformité).

\section{THÉÂTRE DE LA CORRUPTION}

Afin de faciliter l'analyse et pour coller au mieux à la réalité des interactions entre les taxis-motos et les agents de sécurité ou les agents de police, il est possible de présenter ces dernières en trois phases distinctes, à savoir la présentation, la négociation et l'échange d'argent. Précisons qu'avec le renouvellement régulier des taxis-motos, des agents de sécurité et la rotation des agents de police, il est rare que les acteurs se connaissent avant le début de l'interaction, sauf si les agents de sécurité et les taxis-motos sont rattachés à la même coopérative et exercent dans le même quartier. En outre, comme cela a été souligné précédemment, les pratiques de corruption sur les routes de Kigali ne sont plus perpétrées par les mêmes acteurs (les agents de sécurité ayant pris majoritairement la place des policiers dans les activités de contrôle entre 2013 et 2019, même si la police reste bien présente), mais elles persistent en suivant les mêmes formes et les mêmes phases. La socialisation professionnelle et les accointances entre agents de sécurité et agents de police et entre taxis-motos et agents de sécurité sont donc un facteur essentiel dans la reproduction des pratiques présentées ci-dessous.

\section{Scène d'exposition: la corruption de la répression}

Lorsqu'un motard est arrêté, il va invariablement commencer la conversation en implorant le pardon de l'agent de police ou de sécurité dans l'espoir d'obtenir de la clémence. En effet, le motard sait qu'il est perçu comme coupable, indépendamment de toute méconduite. Les motards en appellent à l'indulgence des agents qui personnifient l'autorité et peuvent décider du prix à payer pour la faute du motard: «Comment puis-je te pardonner alors que tu es un pécheur! Je ne peux pas te pardonner comme ça ${ }^{85}$ ! En utilisant un registre religieux et culturel, les taxis-motos pensent obtenir clémence ou pouvoir entrer en négociation. Cette inscription sémantique dans ces registres normalise le processus de négociation, en le souscrivant à une logique d'action et à des codes familiers aux acteurs. Bien entendu, il s'agit aussi d'une rationalité stratégique qui permet aux motards de minimiser le

85. Entretien avec un taxi-moto, Muhuza, 12 octobre 2013. 
coût des amendes et, pour les agents de police ou de sécurité, d'obtenir un apport financier:

«Quand un agent de sécurité t’arrête, il commence à t'accuser de fautes non commises, invraisemblables ou irréelles, il te menace de te conduire devant l'agent de police routière tout près. Quand tu arrives devant l'agent de police, tu ne peux pas t'expliquer, il conduit directement la moto à Kacyiru. De ce fait, le motard commence à vouloir négocier avec cet agent de sécurité de peur de payer une amende de 25000 francs ou plus ${ }^{86}$.»

Lorsqu'Alain, taxi-moto, entend «Tu n'es pas garé correctement! Maintenant qu'est-ce que tu penses? Tu dois te donner des directives ${ }^{87}$ », il sait que les agents de sécurité ou de police sont prêts à négocier. Le tutoiement souligne le rapport de domination des policiers et l'injonction d'agir. Un autre indice est donné lorsqu'il voit que le policier n'ouvre pas son carnet de verbalisation (ou sa tablette en 2019) ou lorsqu'il garde son permis et l'invite à revenir après avoir déposé son passager. Au contraire, si l'agent de police ou de sécurité lui demande de se garer et de libérer le passager, il sait que l'agent n'est pas ouvert à la négociation. Dans les cas (rares) où l'agent de police a clairement indiqué ses intentions mais que le motard refuse de payer le pot-de-vin, le motard prend le risque de se confronter au zèle de l'agent, ce dernier lui faisant alors payer l'amende officielle et inspectant la moto ou les papiers du conducteur pour voir si d'autres amendes peuvent être ajoutées. Ces ratés et ces incompréhensions entre agents et administrés montrent qu'il est fait recours aux normes formelles lorsque les normes informelles ne peuvent guider l'action.

Ces pratiques montrent également l'interdépendance entre les agents et les motards et le développement d'attentes entre les acteurs, au point d'entendre dans plusieurs entretiens cette affirmation: «Un bon policier, c'est celui qui accepte la corruption ${ }^{88}$.» Soulignons également que la peur de la sanction pousse les motards à avoir une préférence pour la corruption, conditionnée à la préférence des agents de sécurité et des policiers. Ainsi, selon l'approche de Bicchieri ${ }^{89}$, les pratiques de corruption des taxis-motos sont un comportement prudentiel, dans le sens où ils défendent leurs intérêts en se conformant aux attentes des agents de police et de sécurité.

L'amorce de la relation est décisive et conditionne le pouvoir de négociation qu'aura chacun des protagonistes par la suite. Les similitudes entre tous les entretiens avec les motards révèlent des règles codifiées et respectées. On peut distinguer une distribution des rôles entre les acteurs où les agents de police

86. Entretien avec un taxi-moto, Nyabugogo, 17 septembre 2019.

87. Entretien avec un taxi-moto, Nyabugogo, 13 août 2019. En kinyarwanda, «donner des directives à quelqu'un» correspond à «kwibwiriza».

88. Entretien avec un taxi-moto, Kicukiro, 9 septembre 2013.

89. C. Bicchieri, Norms in the Wild..., op. cit. 
et de sécurité prennent invariablement l'ascendant sur les motards. Les taxismotos interrogés affirment: «Quand on t’arrête, tu n'as pas de valeur ${ }^{90}$. » Cela corrobore les «croyances» en le pouvoir discrétionnaire des policiers et des agents de sécurité, les deux incarnant l'autorité aux yeux des motards. Il est certain que les agents de police ou de sécurité ont une influence plus grande sur cette première interaction. Certains motards évoquent le comportement inamical de certains agents, y compris des préjugés ou des arrestations arbitraires. D'autres évoquent au contraire des plaisanteries avec les agents, comme un signe ou un moyen d'une potentielle négociation.

Un élément diffère considérablement entre 2013 et 2019: le raisonnement coût-opportunité des protagonistes. En 2013, les motards pouvaient distinguer des «fenêtres d'opportunité» pour la corruption: les policiers étaient plus prompts à se corrompre en fin de journée (c'est-à-dire après 10 heures du matin, lorsque les carnets de verbalisation étaient déjà bien remplis), en fin de mois (lorsque les salaires se font attendre), en négociant avec les subalternes plutôt qu'avec les chefs, et plutôt avec les vétérans qu'avec les jeunes recrues. En 2019, ces distinctions semblent complètement caduques au sein de la police et ne pas s'appliquer aux agents de sécurité des coopératives qui sont «toujours prêts à manger" ${ }^{91}$ ». Cela témoigne d'une évolution des pratiques sur les routes de Kigali où la police semble moins prompte à se corrompre, mais surtout où les opérations de contrôle ont été sous-traitées à des agents de sécurité beaucoup plus corrompus.

\section{L'art de la négociation au cour de l'intrigue}

«Turangyzanye» («finissons-en»). Il s'agit d'une expression utilisée par les agents de police ou de sécurité pour signifier l'entrée en négociation. En 2013, cette dernière a lieu en marge des barrières de police, en 2019, plutôt dans un environnement neutre à l'abri des regards. Pour défendre leurs intérêts, les motards évoquent leurs conditions socio-économiques, ou ce que les policiers et les agents de sécurité peuvent réaliser avec cet «argent gratuit». «Des fois, les agents disent "On va te laisser partir si tu nous donnes 10000 francs rwandais." Que puis-je faire ${ }^{92}$ ?» Durant cette phase, seuls les aspects pécuniers sont évoqués. Les motards n'invoquent pas leur appartenance ethnique ou leur origine géographique, ils considèrent que cela serait une stratégie risquée, certains agents pouvant avoir une réaction d'hostilité en apprenant leurs origines. «Moi,

90. Entretien avec un taxi-moto, Kicukiro, 9 septembre 2013.

91. Entretien avec un taxi-moto, Nyabugogo, 15 août 2019.

92. Entretien avec un taxi-moto, Nyabugogo, 15 décembre 2019. 
je ne dis pas que je viens du Nord, c'est la région du Président Habyarimana ${ }^{93}$.» Ainsi, il y a une attente des acteurs à ce que la conversation reste anonyme. Si cette norme n'est pas respectée, les motards peuvent s'entendre dire «ne joue pas comme ça, tu es coupable, tu dois être pardonné94! »

Les agents de sécurité et de police utilisent parfois des codes linguistiques. Ils peuvent par exemple dire «donne-moi vendredi»; le vendredi étant le cinquième jour de la semaine, cela correspond à 5000 francs rwandais. Les agents peuvent également demander «gutera akantu», littéralement «jeter une petite chose» (2000 francs rwandais), pour oublier l'incident:

"Quand les agents de sécurité t'arrêtent, tu leur paies 1000 ou 2000 francs et ils te laissent partir au lieu de passer du temps à discuter avec eux. Cela est très courant dans notre travail car quand un agent de sécurité t'arrête, il te dit "Gura amazi [achète de l'eau]" et l'affaire est résolue ${ }^{95}$.»

Les agents se montrent souvent explicites en demandant au motard «d'acheter un Fanta» ou de "payer le pardon». Le langage corporel est également utilisé: quatre doigts en l'air signifient 4000 francs rwandais, le poing fermé 5000 francs rwandais. Le fait de donner un sens détourné à des signes usuels ou à des mots en kinyarwanda démontre une volonté de dissimuler des pratiques illicites, de créer une communauté linguistique ${ }^{96}$, une proximité de compréhension des acteurs dans la fonction performative des expressions utilisées.

L'entretien qui suit est assez significatif des manières dont peuvent se nouer les négociations.

\section{Extrait d'entretien avec un taxi-moto ${ }^{97}$}

Moi : À quand remonte ta dernière arrestation?

Damien: Hier, à l'entrée de Nyabugogo, une patrouille a surgi. On voulait me faire payer 50000 francs pour mauvais stationnement, mais j'ai négocié et j'ai payé 10000. Moi : Comment as-tu fait?

Damien: La moto a été embarquée, on m'a dit de me mettre sur le côté. D'autres motos ont été arrêtées et après on a négocié. À la fin, sur le PV, on a écrit que je conduisais avec un client sans casque.

93. Entretien avec un taxi-moto, Nyabugogo, 25 septembre 2013.

94. Ibid.

95. Entretien avec un taxi-moto, Nyakabanda, 27 septembre 2019.

96. W. Labov, «The Exact Description of the Speech Community: Short-a in Philadelphia», in R. W. Fasold et D. Schiffrin (dir.), Language Change and Variation, Amsterdam, Benjamins, 1989, p. 1-57.

97. Entretien avec un taxi-moto, Nyabugogo, 8 septembre 2013. 
Moi : Mais comment s'est faite la négociation?

Damien: Il y a des commissionnaires qui travaillent dans la coopérative, souvent les agents de sécurité. Comme ils connaissent les policiers, ils négocient pour les motards. Moi j'ai appelé mon copain, il a négocié avec le policier et ils sont tombés d'accord. Après j'ai donné l'argent au commissionnaire.

Moi : Comment tu connais ce commissionnaire?

Damien: C'est un copain du parking.

Comme le montre l'extrait ci-dessus, la plupart des pots-de-vin versés constituent de faibles montants. Le montant est toujours proportionnel à l'amende réelle, représentant environ la moitié du prix de l'amende. Par exemple, alors que le port du casque est obligatoire pour des raisons de sécurité, son oubli est formellement sanctionné de 10000 francs rwandais, mais se négocie informellement à 5000 francs rwandais entre les acteurs. La «négociation de l'État ${ }^{98}$ », en l'occurrence ici le marchandage des montants établis pour les infractions au Code de la route par les agents de police et de sécurité, montre le lien étroit entre le cadre formel et les pratiques informelles, où le pragmatisme des agents les pousse à proposer un prix sur la base d'un critère officiel pour juger la faute commise, tout en considérant un coût acceptable pour les taxis-motos et en incorporant le risque encouru. Soulignons en effet que le risque de sanctions pour les agents reste bien réel puisque des condamnations à un ou deux ans de prison sont prononcées même pour des pots-de-vin aussi faibles que 1000 ou 2000 francs rwandais ${ }^{99}$.

Un autre aspect intéressant dans l'entretien ci-dessus est le rôle de l'intermédiation, une notion importante dans l'ethnographie de l'État africain pour comprendre les logiques sociales à l'œuvre dans le déploiement de l'action publique $^{100}$. Lorsque la moto est saisie ou lorsque le montant de l'amende devient trop élevé, les acteurs peuvent s'appuyer sur des intermédiaires pour sécuriser la transaction, faciliter les démarches et un arrangement à l'amiable. Un intermédiaire peut être un membre de la parentèle, un représentant de la coopérative ou quiconque dans le réseau de référence des acteurs qui va se faire rétribuer pour garantir les termes de la négociation. Ce processus d'intermédiation fait écho à ce que Baez Camargo et Koechlin nomment les pratiques de «camouflage» permettant de protéger et de légitimer le réseau social et ses pratiques en insérant l'action dans des pratiques usuelles ${ }^{101}$.

98. T. Bierschenk et J.-P. Olivier de Sardan (dir.), States at Work..., op. cit.

99. Republic of Rwanda, Ombudsman, Corruption Convicts Database 2013, 2014, 2015.

100. G. Blundo, «Négocier l'État au quotidien: agents d'affaires, courtiers et rabatteurs dans les interstices de l'administration sénégalaise», Autrepart, n²0, 2001, p. 75-90.

101. C. Baez Camargo et L. Koechlin, «Informal Governance... », art. cité. 
Comme le souligne Cissokho ${ }^{102}$, l'intermédiation est également un moyen de conserver l'apparence d'un «fonctionnement bureaucratique idéal» (si cher à l'État rwandais) tout en détournant discrètement dans la pratique le sens de cet idéal-type wébérien du fonctionnaire.

Enfin, dans l'entretien ci-dessus, Damien précise que cet intermédiaire est un «copain du parking». Cela montre le rôle essentiel de la socialisation entre les taxis-motos et les agents de sécurité dans le maintien des pratiques de corruption. Si la négociation est possible entre taxis-motos et agents de sécurité qui ne se connaissent pas, elle est plus facile entre personnes qui se côtoient sur les parkings ou au sein des coopératives. Il arrive même qu'ils donnent spontanément un "petit quelque chose» («akanu») aux agents de sécurité de leur parking afin de faciliter une prochaine négociation. En revanche, cette familiarité n'existe pas lorsque les taxis-motos exercent en dehors de leur quartier, ce qui accroît le risque de ne pas réussir à négocier. Les normes empiriques apparaissent alors essentielles pour sécuriser les négociations et maintenir les pratiques du milieu.

\section{Dénouement: l'échange d'argent}

À ce stade de l'interaction, les risques sont plus importants et la priorité des acteurs est de sécuriser la transaction en la dissimulant. Soulignons que ce besoin de dissimulation est moindre en 2019 qu'en 2013 puisque les contrôles de sécurité sont plus fréquemment effectués par les agents de sécurité et que ces derniers sont moins précautionneux que les agents de police. Tout d'abord, l'échange d'argent est différé le temps que le motard le trouve (de quelques heures à quelques jours). Ensuite, avec la police, les motards doivent se présenter en habit civil (sans le gilet officiel des taxis-motos et le casque). Pour un simple pot-de-vin, le motard peut mettre l'argent dans la paume de sa main et serrer la main de l'agent de sécurité ou de police, qui empoche ensuite discrètement l'argent. Les acteurs peuvent également s'entendre sur le transfert de l'argent via une application mobile ou avoir recours à une personne tierce, comme un colporteur dans la rue, par exemple en achetant du crédit téléphonique.

En général, les agents de sécurité patrouillent par pair, partageant les responsabilités (parfois l'un fait le guet pendant que l'autre empoche l'argent), les risques de la transaction ainsi que les gains. Cette solidarité crée un

S. Cissokho, «Petits échanges entre amis. Remarques sur l'ambivalence des rapports routiniers entre fonctionnaires et usagers au Sénégal ", Revue française de science politique, vol. 67, n 4, 2017, p. 631-651. 
effet-miroir qui normalise les pratiques de corruption et permet de renforcer les valeurs communes des agents de sécurité tout en minimisant le risque de trahison au sein des équipes. Le non-partage du butin est mal perçu et sanctionné, ce qui souligne les attentes empiriques et normatives des agents ${ }^{103}$.

Dans le jargon, lorsqu'un motard se fait arrêter et réussit à corrompre un policier, il narrera plus tard à ses collègues qu'il s'est fait «couper la queue » («gucika umurizo»), ce qui signifie qu'il a dû partager ses gains avec quelqu'un d'autre. Il peut également parler de «cinq bâtons» («inkoni eshanu») pour évoquer les cinq coups de bâtons (5000 francs rwandais) donnés par les agents, ou dire «l'État m'a frappé». À travers ces expressions, la corruption est assimilée à une perte, une infortune, mais aussi à une injustice sociale en lien avec ce qu'ils considèrent comme un châtiment illégitime. Pour reprendre l'analyse sémiologique de Blundo et Olivier de Sardan, il s'agit ici d'un registre basé sur l'extorsion entre un prédateur et une victime ${ }^{104}$.

Pour une majorité de motards, la corruption est autant un vol (de leur argent) qu'une faute morale, mais aussi une nécessité: «La corruption c'est mal, mais cela nous permet de continuer à travailler ${ }^{105}$.» Les entretiens montrent que la rationalité économique prévaut sur les raisonnements éthiques, même si les motards se disent tristes que leur argent aille dans les poches des agents plutôt qu'à la collectivité. Soulignons que les motards sont des contribuables importants: avec 72000 francs rwandais d'impôt forfaitaire annuel sur les bénéfices, les 32000 taxis-motos rapportent plus de 2,3 milliards de francs rwandais par an. Les pots-de-vin ne peuvent donc pas être considérés comme une forme de défiance face à une injonction d'imposition, mais bien une opportunité d'éviter de payer des amendes considérées comme trop élevées ou illégitimes (encore plus lorsqu'elles sont collectées par les agents de sécurité). Ainsi les motards évoquent la corruption comme un recours légitime pour défendre leurs propres intérêts.

$\boldsymbol{S}_{i}$ la corruption de la police routière a diminué sur la période étudiée, les pratiques de subornation sur les routes de Kigali restent présentes et se sont mêmes pérennisées nonobstant le strict encadrement légal et procédural. Cela souligne l'impact relatif de la politique de tolérance zéro contre la corruption, qui semble se diluer au gré du contexte normatif et social et des relations de pouvoir entre les acteurs. L'État rwandais est «négocié» au quotidien par les détenteurs de l'autorité, qui ont la volonté et la capacité 
de faire valoir leurs intérêts personnels. Cette négociation informelle de l'État, cette imbrication du formel et de l'informel font écho à de nombreuses situations similaires dans d'autres pays africains. Cela montre les limites de l'exceptionnalisme rwandais. Le hiatus est d'ailleurs saisissant entre d'un côté cette image singulière projetée par le régime «d'hypermodernité » et de contrôle, et de l'autre la résilience de pratiques et de «bricolages » qui font partie du quotidien des Rwandais et qui contredisent cette image, et par là même les projets gouvernementaux. Les mesures anti-corruption sont ainsi instrumentalisées pour servir l'image du pays, sans que cela ne se traduise dans les faits par une remise en cause de certaines pratiques (favoritisme ou abus de pouvoir).

L'originalité de l'étude est de montrer que ce contexte «autoritaire » conduit finalement à une application partiale des règles sans que cette partialité puisse être contestée puisqu'elle fait partie de cette verticalité du pouvoir au Rwanda. Dans ce contexte, l'autorégulation des agents (ici les coopératives) peut induire des pratiques de corruption qu'il est très difficile pour les acteurs de signaler ou de contrer. La particularité rwandaise réside ainsi dans cette imposition d'un contrôle qui se veut étroit, s'appuyant tant sur le cadre formel qu'informel, auprès de structures qui ne sont redevables qu'envers le haut. En ajoutant de nouvelles couches administratives de supervision sans transparence ni redevabilité sociale, l'État génère de nouvelles opportunités pour les pratiques de corruption. Ainsi, cet enchâssement social des politiques publiques au Rwanda - cette coproduction de l'ordre - engendre une coproduction de la corruption. Prenant la forme d'un cercle vicieux, le déploiement d'une politique de tolérance zéro vis-à-vis de la corruption s'appuie sur et alimente cette verticalité du pouvoir, masquant et finalement perpétuant certaines pratiques de malversation intrinsèques à la gouvernance rwandaise.

Enfin, l'étude a permis de mieux définir la nature et les déclencheurs de la corruption au Rwanda. L'approche par les normes sociales s'est révélée très utile pour observer les attentes réciproques des acteurs. Elle a permis d'écarter l'idée de la corruption comme norme sociale dans le milieu étudié, les attentes normatives n'étant pas la cause des pratiques observées. Les connivences, les rapports de force, l'arbitrage coût-opportunité s'avèrent en revanche des déterminants essentiels de la corruption, encore plus dans le contexte de sous-traitance de l'autorité formelle auprès de structures ayant une gestion opaque. Le langage utilisé, les postures des agents, l'échange d'argent forment un savoir-faire qui se transmet à travers une socialisation dans le milieu professionnel alors même que les agents impliqués changent. Finalement, les pots-de-vin et les pratiques de favoritisme ou de clientélisme vont bien au-delà d'un abus de pouvoir légitime à des fins de profits 
personnels; ils révèlent des logiques sociales et économiques qui sont à la fois des moyens sur lesquelles s'appuie le régime rwandais qu'une partie des résultats de sa gouvernance actuelle

Guillaume Nicaise

Chr. Michelsen Institute,

U4 Anti-Corruption Resource Centre

\begin{abstract}
On the Roads of Kigali: Evolution of Petty Corruption Practices

The fight against corruption increased the informal privatization of the management of the road transport sector in Rwanda, to the benefit of transport cooperatives, which replaced traffic police. This interweaving of formal and informal governance encourages the misuse of rules on public roads. Corruption is thus facilitated by a lack of control over security agents' practices and multiple relations of domination against motorcycle taxis. The authoritarian context of Rwanda ultimately leads to a partial application of the rules, which cannot be contested. While the contrast between the image of Rwanda and the empirical reality is striking, the functioning of the state shows many similarities with other African countries, questioning the idea of Rwandan exceptionalism.
\end{abstract}

